\title{
Non-Communicable Diseases (NCDs) and Injuries Director Ministry of Public Health Kabul Afghanistan
}

\author{
Said Habib Arwal* \\ Ministry of Public Health, Kabul, Afghanistan \\ *Corresponding author: Said Habib Arwal, Community Based Health Care National Coordinator, Ministry of Public Health, Kabul, Afghanistan \\ Submission: 望 November 10, 2017; Published: 眥 January 22, 2018
}

\section{Introduction}

Evidence, including from the Afghanistan mortality survey (AMS), identifies the increasing mortality due to the major NCDs and injuries, which have a major impact on the health of communities, the health system, and the economy of Afghanistan, as a major challenge.

The determinants of non-communicable diseases are multifactorial and often are outside the control and influence of the health sector. The four main groups of non-communicable diseases share four main risk factors: tobacco use, unhealthy diet, harmful use of alcohol and insufficient physical activity. To reduce these risk factors, changes in the lifestyles and behaviors of individuals and families is necessary. Because of the communal nature of the Afghan society, there is a need for interventions in community settings to be at mosques, villages, schools or workplaces, to promote and encourage healthy behaviors to prevent non-communicable diseases. Moreover, the prevention, detection and management of non-communicable diseases are important to decrease suffering and reduce deaths from the mentioned diseases. These all can be done through a coordinated multi-sectoral national response.

The Afghan Ministry of Public Health as the steward of health sector, has assumed the responsibility to tackle the burden of non-communicable diseases, in view of the demographic and epidemiologic transition envisaged in the near future. The MoPH has taken some steps toward initiating a comprehensive response to non-communicable diseases. Establishment of the Department of Non-communicable Diseases within the structure of the MoPH is an important first step in this regard. The department, since its initiation, has prepared a vision to tackle non-communicable diseases through evidence-based interventions adapted to the local context and situation. Development of a national non-communicable diseases strategy is an indispensable step, which provides clear guidance to the department on priority interventions and actions to take in order to reduce the burden of these diseases and improve the quality of life for the people of Afghanistan. The interventions outlined in this document will guide many public and private sector individuals and organizations involved in the prevention, and control of non-communicable diseases.

Having a National NCDs Strategy and Key Strategic focus areas supported by Action Plans, is essential for ensuring that strategies and guidelines are in place for the prevention and control of noncommunicable diseases in Afghanistan.

Government of Afghanistan and the Ministry of Public Health's commitment to regional and international initiatives such as:

A. The Political Declaration of the High-Level Meeting of the UN General Assembly on the Prevention and Control of Noncommunicable Diseases, September 2011

B. The Global Action Plan for the Prevention and Control of Non-communicable Diseases 2013-2020, approved by the World Health Assembly in May 2013

C. Other WHO related strategies and plans of action (Tobacco , Nutrition, etc)

D. Alma Ata and Primary Health Care Declaration 1978

\section{Background}

Non-communicable diseases (NCDs) consist of a vast group of non-infectious medical conditions, but in terms of premature mortality, emphasis has been on cardiovascular disease, cancer, diabetes, and chronic respiratory diseases. Although NCDs were not in the global agenda when the United Nations developed the Millennium Development Goals (MDGs), however it has raised concerns and has culminated in the United Nations (UN) resolution on Political Declaration of the High-level Meeting of the General Assembly on the Prevention and Control of Non-communicable Diseases in September 2011. This special session placed NCDs on the global development agenda [1]. Later on in 2012, the World Health Assembly endorsed an important new health goal to reduce 
avoidable mortality from NCDs by $25 \%$ by 2025 which is called the 25 by 25 goal [2]. To achieve the target of $25 \%$ reduction in preventable deaths health systems and strategic framework need to be transformed to provide sufficient care for both communicable and NCDs in Afghanistan.

\section{Global Health and Economic Burden}

In 1990, there were 26.6 million deaths worldwide from NCDs increasing in 2010 to 34.5 million. Likewise the global burden of NCDs has increased from $43 \%$ (1.08 billion of the total 2.50 billion) in 1990 , to $54 \%$ (1.34 billion of 2.49 billion) of the total number of disability-adjusted life-years in 2010. Furthermore in 2008, around 57 million deaths were reported globally, of which 36 million (or $63 \%$ ) were due to NCDs. The most common NCDs contributed to global deaths were cardiovascular diseases, diabetes, cancer and chronic respiratory diseases. Of the total NCDs deaths globally, nearly $80 \%$ occur in low-and middle income countries. Likewise the global economic burden of NCDs is large, estimated at US\$6.3 trillion in 2010, rising to $\$ 13$ trillion in 2030. A $10 \%$ rise in NCDs leads to a $0.5 \%$ decrease in gross domestic product [3].

Due to quick demographic and epidemiological transitions in South Asia life expectancy is increasing and fertility rate is reducing which has leaded to increased health burden of NCDs with 46 percent (55 percent including injuries) of burden of disease as a proportion of total forgone DALYs. It has been indicated that prevalence of smoking varies in South Asian countries from 16$32 \%$, alcohol consumption between $3-41 \%$, eating less than 5 servings of fruits and vegetable $81-99 \%$, physical inactivity $4-24 \%$, overweight and obesity $9-44 \%$, raised blood pressures $8-42 \%$, raised fasting blood sugar 4-9\%, and raised blood cholesterol 13$54 \%$. In neighboring Iran a systematic analysis of studies from 1996 to 2004 estimated that the overall prevalence of hypertension in 30-55 and $>55$-year-old population were around $23 \%$ and $50 \%$, respectively. The prevalence in men was $1.3 \%$ less than that in women. In a study in Pakistan eastern neighboring country the overall prevalence of hypertension was $26 \%$, with differentiation in males (34\%) versus females (24\%). Age analysis revealed that the prevalence of hypertension increased with age and hypertensive subjects were 5.6 times more likely to be over 35 years of age.

\section{Burden of NCDs in Afghanistan}

As Afghanistan is in the early stages of the demographic transition therefore the burden of NCD is adding up to the ongoing burden of communicable diseases, causing a double disease burden on the population. The transition will become more evident in the years to come. According to predictions the proportion of the population 65 years and older will increase from 2.1 percent, in 2000 , to 2.9 percent, in 2025. Source As older populations are more likely to be affected by NCDs, the health burden from NCDs will rise in parallel with population aging.

In 2004, NCDs (inclusive of injuries) accounted for 46 percent in terms of the number of lives lost due to ill health, disability, and early death (DALYs), with the remainder from communicable diseases and maternal and child health issues.

Due to less attention to NCDs and years of conflict information of burden of morbidity and mortality contributed by NCDs is not fully available in Afghanistan. Nonetheless, the total DALYs forgone attributed to NCDs in Afghanistan is estimated to be $43 \%$. Afghanistan Mortality Survey (AMS) 2010 revealed that $33.3 \%$ of all deaths in Afghanistan are attributed to NCDs compare to $42.6 \%$ of all together cause of death due to communicable, maternal, prenatal, and nutrition conditions. Cardiovascular diseases, malignant neoplasm, diabetes, respiratory diseases, and digestive diseases are the leading cause of deaths due to NCDs, which account $14 \%, 7.3 \%, 3.7 \%, 1.9 \%$, and $1.8 \%$ of total NCDs deaths, respectively.

Based on a World Health Organization (WHO) estimates, 2000there were 468,000 people with diabetes in Afghanistan. This number is expected to rise to $1,403,000$ in 2030, representing nearly a threefold increase when compared to 2000 . The prevalence of Diabetes among population of 20-79 years have been estimated for Afghanistan in which according to adjustment of national population the prevalence is $6.6 \%$ and $7 \%$ for 2010 and 2030, however after the world population adjustment the prevalence for 2010 is estimated $8.6 \%$ and for 2030 it is projected to be $9.9 \%$.

Likewise little is known about the prevalence of risk factors of NCDs in Afghanistan, yet. However in a study among men 15 years and older in Kabul city revealed that the prevalence of smoking is $35 \%$ Moreover in a study with the aim of identifying the prevalence and risk factors of NCDs among adult population ( $\geq 40$ years) in urban portion of Kabul city in 2012 , it was revealed that the prevalence of Diabetes Mellitus was $13.27 \%$ (obesity with $\mathrm{BMI} \geq 30$ was $31.2 \%$ and prevalence of hypertension was $46.2 \%$ . The prevalence of factors among study subjects were: current smoking (5\%), snuff users (8.9\%), using solid fat in kitchen (69\%), frequency of taking 3 times or less vegetable and fruits per week (58\%). In addition, an assessment of air quality of Kabul city revealed the ambient air quality in the city has deteriorated to such extend that it can be ranked among the dirtiest cities in the world which potentially increase the burden of respiratory diseases and different types of cancer among human .

\section{Conclusion}

A. Evidence, including from the Afghanistan mortality survey (AMS), identifies the increasing mortality due to the major NCDs and injuries, which have a major impact on the health of communities, the health system, and the economy of Afghanistan, as a major challenge.

B. Government of Afghanistan and the Ministry of Public Health's commitment to regional and international initiatives such as:

i. The Political Declaration of the High-Level Meeting of the UN General Assembly on the Prevention and Control of Noncommunicable Diseases, September 2011 
ii. The Global Action Plan for the Prevention and Control of Non-communicable Diseases 2013-2020, approved by the World Health Assembly in May 2013

iii. Other WHO related strategies and plans of action ( DPAS, Tobacco , Nutrition, etc)

iv. Alma Ata and Primary Health Care Declaration 1978

C. Non-communicable diseases (NCDs) consist of a vast group of non-infectious medical conditions, but in terms of premature mortality, emphasis has been on cardiovascular disease, cancer, diabetes, and chronic respiratory diseases.

D. Global Health and Economic Burden, In 1990, there were 26.6 million deaths worldwide
E. Burden of NCDs in Afghanistan: As Afghanistan is in the early stages of the demographic transition therefore the burden of NCD is adding up to the ongoing burden of communicable diseases, causing a double disease burden on the population. The transition will become more evident in the years to come.

\section{References}

1. National Strategy for Prevention and Control of Non-communicable diseases (NCDs) Ministry of public Health Afghanistan, 2015 -2020.

2. Afghanistan mortality survey (AMS).

3. MoPH NCDI Department Monitoring Documents. 